\section{SOI: $1.1 /$ TAS DOI: $10.15863 /$ TAS International Scientific Journal Theoretical \& Applied Science}

p-ISSN: 2308-4944 (print) e-ISSN: 2409-0085 (online)

Year: 2017 Issue: 08 Volume: 52

Published: $20.08 .2017 \quad$ http://T-Science.org
Denis Chemezov

Master of Engineering and Technology, Corresponding Member of International Academy of Theoretical and Applied Sciences, Lecturer of Vladimir Industrial College, Russian Federation chemezov-da@yandex.ru

SECTION 7. Mechanics and machine construction.

\title{
STRESS-STRAIN STATE OF METALLIC ALLOYS AT DIFFERENT TEMPERATURES
}

\author{
Abstract: The dependencies of stress from deformation of steels, irons and non-ferrous alloys in conditions of \\ exposure to thermal loads are presented in the article. \\ Key words: stress, deformation, alloy, temperature, dependence. \\ Language: English \\ Citation: Chemezov D (2017) STRESS-STRAIN STATE OF METALLIC ALLOYS AT DIFFERENT \\ TEMPERATURES. ISJ Theoretical \& Applied Science, 08 (52): 5-11. \\ Soi: http://s-0-i.org/1.1/TAS-08-52-2 Doi: crossef https://dx.doi.org/10.15863/TAS.2017.08.52.2
}

\section{Introduction}

Under the exposure of external loads different materials (in particular metallic alloys) of the machine parts are exposed to elastic or plastic deformations [1]. Stresses occur in the materials which under sustained static or imposed dynamic loads lead toward partial fracture of the part $[2 ; 3 ; 4$; $5 ; 6 ; 7]$. Since most machine parts are mated among themselves by the surfaces and are operated at high speeds, then it is necessary to identify stress-strain state of the materials taking into account the existing thermal loads on them.

\section{Materials and methods}

Calculation of the stress value in different structural alloys from deformation was implemented in the computer program LVMFlow. Following metallic alloys were taken for the research [8]: carbon steel $16 \mathrm{MnCr} 5(\mathrm{NBN})$, alloy steel SCMnCr3 (JIS), corrosion-resistant steel X10CrNiTi18-9 (DIN), grey cast iron EN-GJL-200 (EN), malleable cast iron EN-GJS-700 (EN), brass CuZn38Pb1.5 (DIN), without tin bronze CC330G (EN), aluminium foundry alloy G-AlSi11 (DIN) and zinc alloy ZA-12. Young's modulus of researched alloys at different temperatures: carbon steel $16 \mathrm{MnCr} 5-211.4 \mathrm{GPa} / 1$ ${ }^{\circ} \mathrm{C}, 206.9 \mathrm{GPa} / 100{ }^{\circ} \mathrm{C}, 199.6 \mathrm{GPa} / 200{ }^{\circ} \mathrm{C}, 191.7$ $\mathrm{GPa} / 300{ }^{\circ} \mathrm{C}, 183.4 \mathrm{GPa} / 400{ }^{\circ} \mathrm{C}, 174.9 \mathrm{GPa} / 500$ ${ }^{\circ} \mathrm{C}, 166.1 \mathrm{GPa} / 600{ }^{\circ} \mathrm{C}, 105.7 \mathrm{GPa} / 1428{ }^{\circ} \mathrm{C}$; alloy steel SCMnCr3 $-207.51 \mathrm{GPa} / 25^{\circ} \mathrm{C}, 181.29 \mathrm{GPa} /$
$390{ }^{\circ} \mathrm{C}, 159.56 \mathrm{GPa} / 670{ }^{\circ} \mathrm{C}, 149.52 \mathrm{GPa} / 713.52$ ${ }^{\circ} \mathrm{C}, 138.14 \mathrm{GPa} / 773.86{ }^{\circ} \mathrm{C}, 135.97 \mathrm{GPa} / 800{ }^{\circ} \mathrm{C}$, $119.31 \mathrm{GPa} / 1000{ }^{\circ} \mathrm{C}, 111.78 \mathrm{GPa} / 1090{ }^{\circ} \mathrm{C}$; corrosion-resistant steel X10CrNiTi18-9 $-209 \mathrm{GPa} /$ $20{ }^{\circ} \mathrm{C}, 196.49 \mathrm{GPa} / 200{ }^{\circ} \mathrm{C}, 181.47 \mathrm{GPa} / 400{ }^{\circ} \mathrm{C}$, $165.16 \mathrm{GPa} / 600{ }^{\circ} \mathrm{C}, 150.09 \mathrm{GPa} / 800{ }^{\circ} \mathrm{C}, 116.99$ $\mathrm{GPa} / 1200{ }^{\circ} \mathrm{C}, 104.6 \mathrm{GPa} / 1310{ }^{\circ} \mathrm{C}, 0.5 \mathrm{GPa} / 1455$ ${ }^{\circ} \mathrm{C}$; grey cast iron EN-GJL-200 $-100 \mathrm{GPa} / 1{ }^{\circ} \mathrm{C}, 84$ $\mathrm{GPa} / 100{ }^{\circ} \mathrm{C}, 82.1 \mathrm{GPa} / 200{ }^{\circ} \mathrm{C}, 80 \mathrm{GPa} / 300{ }^{\circ} \mathrm{C}$, $78 \mathrm{GPa} / 400{ }^{\circ} \mathrm{C}, 75.7 \mathrm{GPa} / 500{ }^{\circ} \mathrm{C}, 50 \mathrm{GPa} / 1145$ ${ }^{\circ} \mathrm{C}, 0.5 \mathrm{GPa} / 1168{ }^{\circ} \mathrm{C}$; malleable cast iron EN-GJS$\underline{700}-164 \mathrm{GPa} / 50{ }^{\circ} \mathrm{C}, 159 \mathrm{GPa} / 150{ }^{\circ} \mathrm{C}, 154 \mathrm{GPa} /$ $250{ }^{\circ} \mathrm{C}, 148 \mathrm{GPa} / 350{ }^{\circ} \mathrm{C}, 139 \mathrm{GPa} / 500{ }^{\circ} \mathrm{C}, 82.5$ $\mathrm{GPa} / 1153{ }^{\circ} \mathrm{C}, \quad 0.5 \mathrm{GPa} / 1180{ }^{\circ} \mathrm{C}$; brass $\underline{\mathrm{CuZn} 38 \mathrm{~Pb} 1.5}-97 \mathrm{GPa} / 100{ }^{\circ} \mathrm{C}, 0.5 \mathrm{GPa} / 925^{\circ} \mathrm{C}$; without tin bronze CC330G $-110 \mathrm{GPa} / 100{ }^{\circ} \mathrm{C}, 0.5$ $\mathrm{GPa} / 1045^{\circ} \mathrm{C}$; aluminium foundry alloy G-AlSi11 $70 \mathrm{GPa} / 100{ }^{\circ} \mathrm{C}, 0.5 \mathrm{GPa} / 590^{\circ} \mathrm{C}$; zinc alloy ZA-12 - $133 \mathrm{GPa} / 100{ }^{\circ} \mathrm{C}, 0.5 \mathrm{GPa} / 435^{\circ} \mathrm{C}$. Maximum deformation of all alloys was taken as value of 0.2 .

\section{Results and discussion}

The dependencies of stresses in considered alloys from deformation when exposed to temperature are presented in Figs. 1 - 9.

All metal alloys are characterized by decrease of stress when the temperature is increased. Given the same value of deformation, carbon steel $16 \mathrm{MnCr} 5$ in solid state and at small temperatures may be exposed by significant stresses. 


\begin{tabular}{|c|c|c|c|c|c|c|}
\hline Impact Factor: & $\begin{array}{l}\text { ISRA (India) } \\
\text { ISI (Dubai, UAE } \\
\text { GIF (Australia) } \\
\text { JIF }\end{array}$ & $\begin{array}{r}=1.344 \\
=0.829 \\
=0.564 \\
=1.500\end{array}$ & $\begin{array}{l}\text { SIS (USA) } \\
\text { PИНЦ (Russia) } \\
\text { ESJI (KZ) } \\
\text { SJIF (Morocco) }\end{array}$ & $\begin{array}{l}=0.912 \\
=0.207 \\
=\mathbf{3 . 8 6 0} \\
=\mathbf{2 . 0 3 1}\end{array}$ & $\begin{array}{l}\text { ICV (Poland) } \\
\text { PIF (India) } \\
\text { IBI (India) }\end{array}$ & $\begin{array}{l}=6.630 \\
=1.940 \\
=4.260\end{array}$ \\
\hline
\end{tabular}

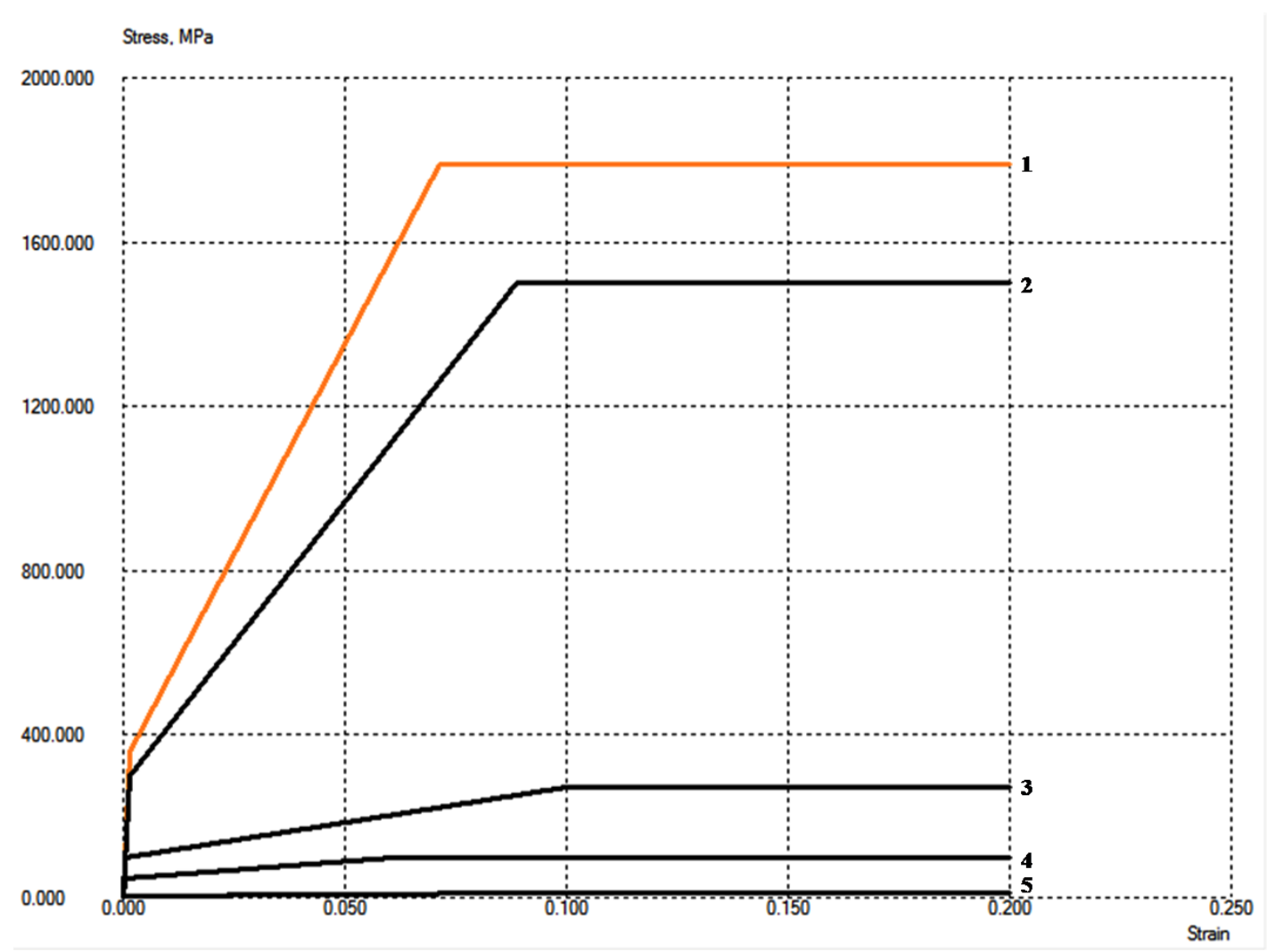

Figure 1 - The dependencies of stress in carbon steel $16 \mathrm{MnCr} 5$ from deformation: 1) $\mathrm{T}$ (temperature) $=1{ }^{\circ} \mathrm{C}$, 2) $\left.\left.\left.\mathrm{T}=100{ }^{\circ} \mathrm{C}, 3\right) \mathrm{T}=600^{\circ} \mathrm{C}, 4\right) \mathrm{T}=1000{ }^{\circ} \mathrm{C}, 5\right) \mathrm{T}=1450{ }^{\circ} \mathrm{C}$.

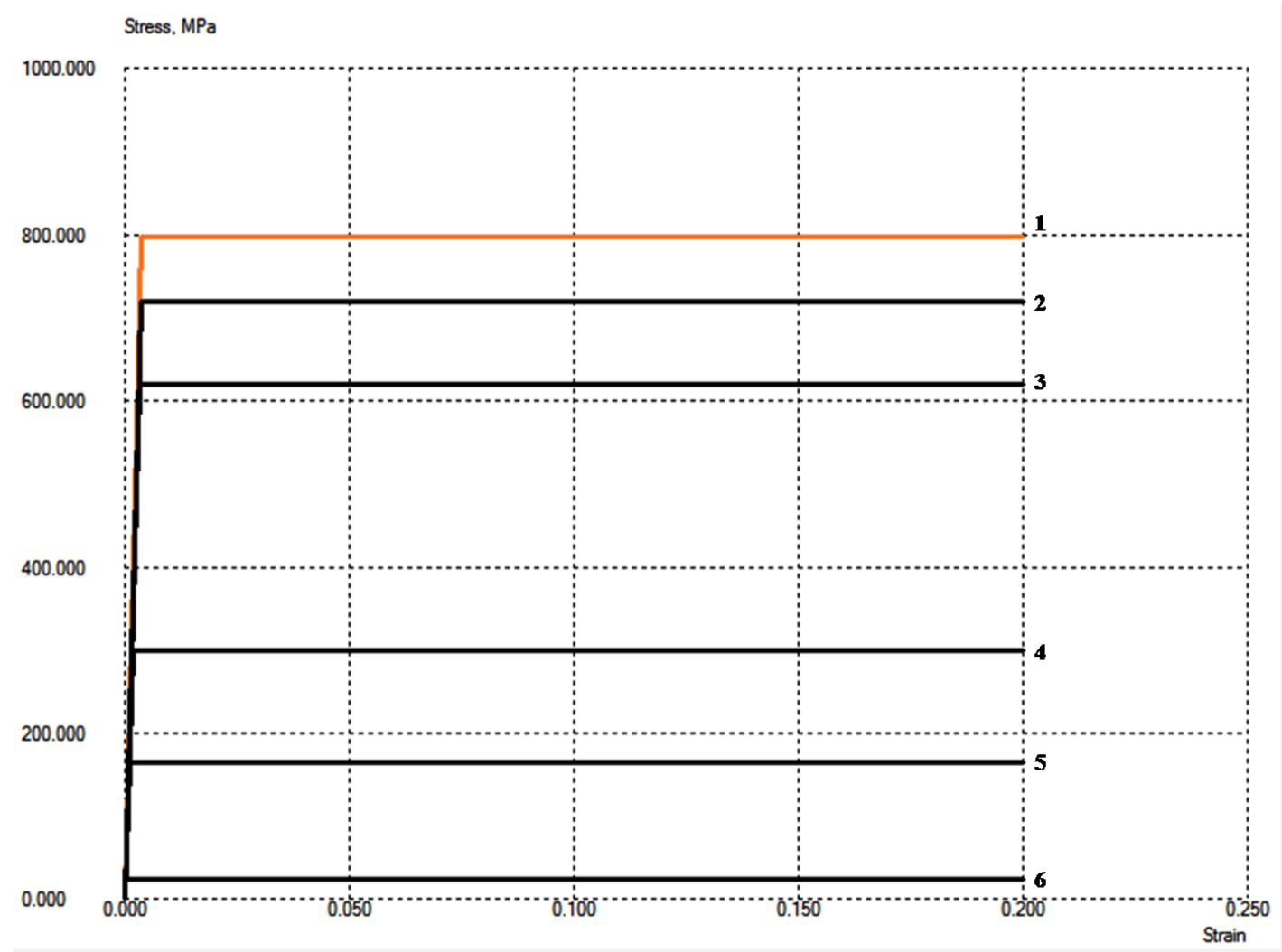

Figure 2 - The dependencies of stress in alloy steel SCMnCr3 from deformation: 1) $\left.\mathrm{T}=25^{\circ} \mathrm{C}, 2\right) \mathrm{T}=200{ }^{\circ} \mathrm{C}$, 3) $\mathrm{T}=400{ }^{\circ} \mathrm{C}$, 4) $\left.\left.\mathrm{T}=600{ }^{\circ} \mathrm{C}, 5\right) \mathrm{T}=800{ }^{\circ} \mathrm{C}, 6\right) \mathrm{T}=1090{ }^{\circ} \mathrm{C}$. 


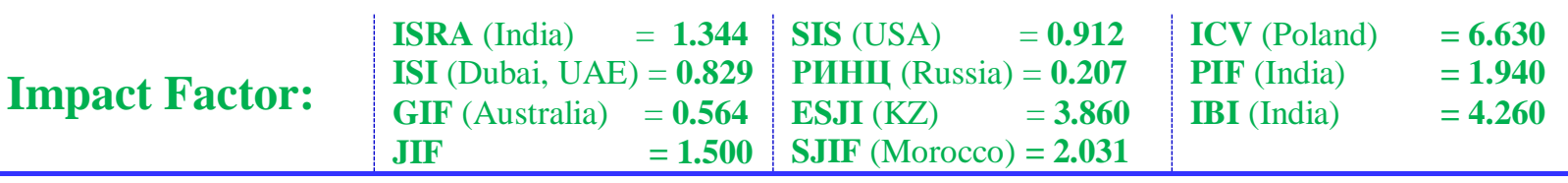

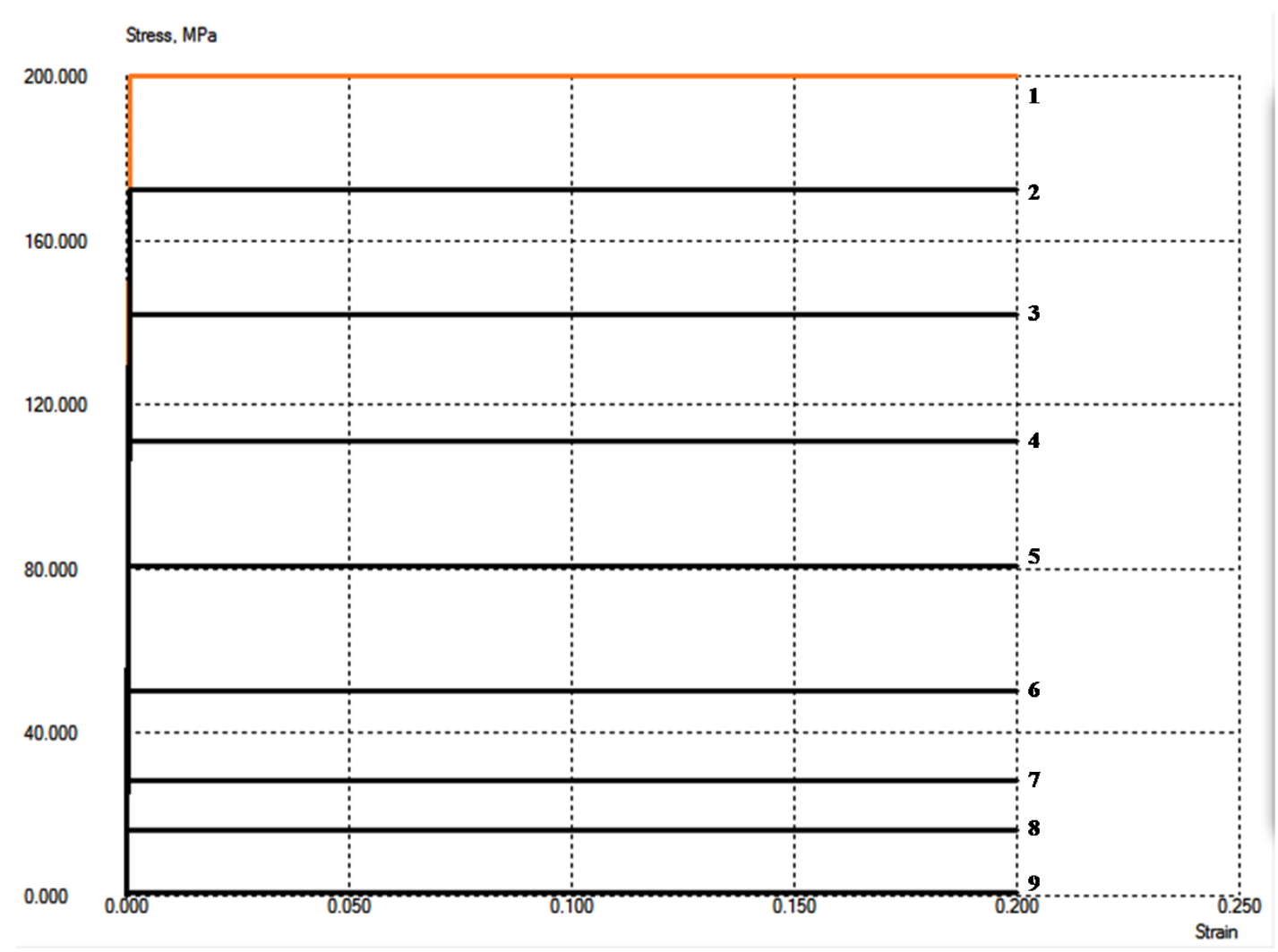

Figure 3 - The dependencies of stress in corrosion-resistant steel X10CrNiTi18-9 from deformation: 1) $\mathrm{T}=$ $20{ }^{\circ} \mathrm{C}$, 2) $\left.\left.\left.\left.\left.\left.\mathrm{T}=200{ }^{\circ} \mathrm{C}, 3\right) \mathrm{T}=400{ }^{\circ} \mathrm{C}, 4\right) \mathrm{T}=600^{\circ} \mathrm{C}, 5\right) \mathrm{T}=800^{\circ} \mathrm{C}, 6\right) \mathrm{T}=1000{ }^{\circ} \mathrm{C}, 7\right) \mathrm{T}=1200{ }^{\circ} \mathrm{C}, 8\right) \mathrm{T}=1310$ ${ }^{\circ} \mathrm{C}$, 9) $\mathrm{T}=1455^{\circ} \mathrm{C}$.

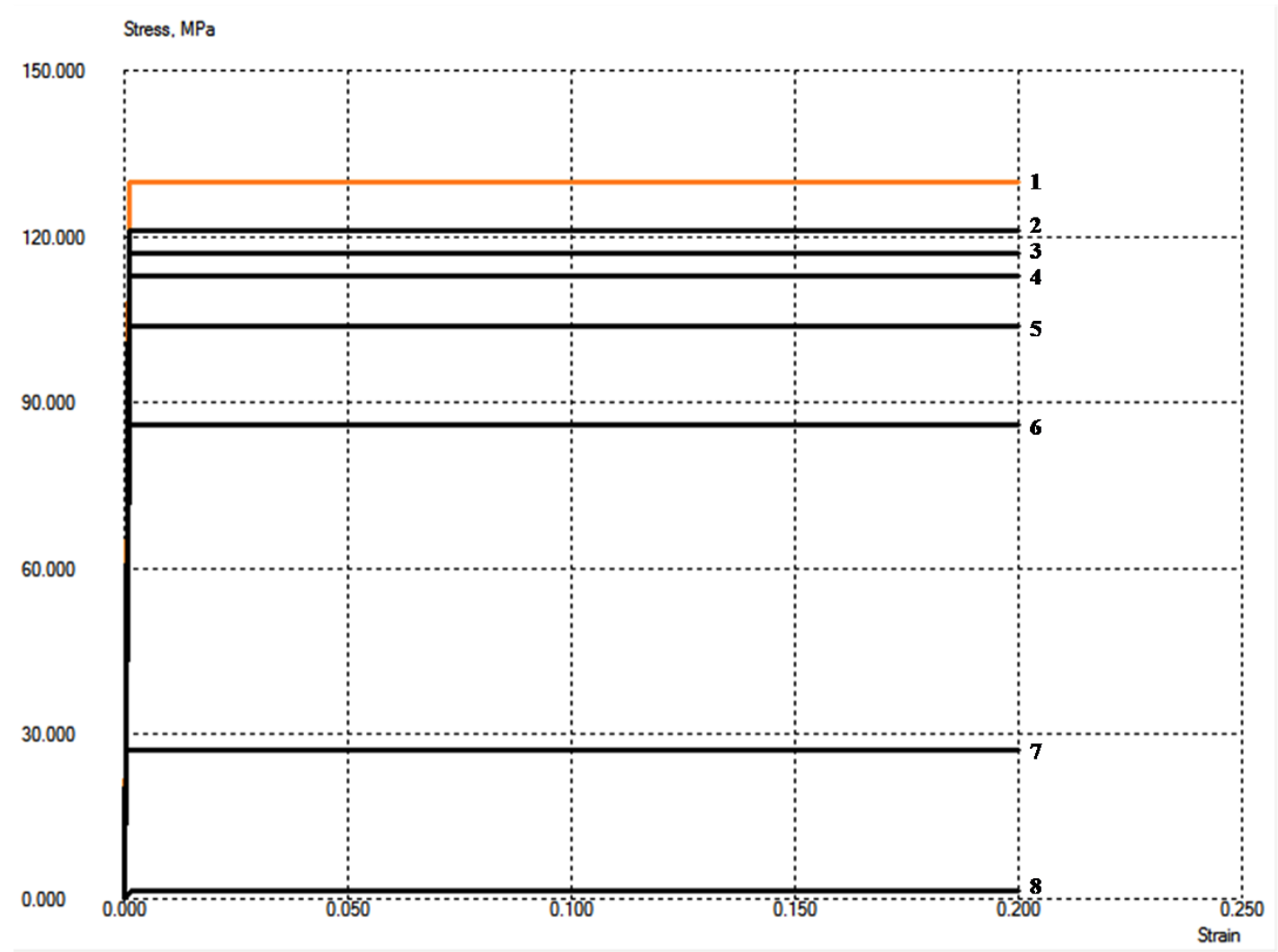

Figure 4 - The dependencies of stress in grey cast iron EN-GJL-200 from deformation: 1) $\left.\mathrm{T}=20^{\circ} \mathrm{C}, 2\right) \mathrm{T}=$ $\left.\left.\left.\left.\left.\left.100{ }^{\circ} \mathrm{C}, 3\right) \mathrm{T}=200{ }^{\circ} \mathrm{C}, 4\right) \mathrm{T}=300{ }^{\circ} \mathrm{C}, 5\right) \mathrm{T}=400{ }^{\circ} \mathrm{C}, 6\right) \mathrm{T}=500{ }^{\circ} \mathrm{C}, 7\right) \mathrm{T}=1000{ }^{\circ} \mathrm{C}, 8\right) \mathrm{T}=1168{ }^{\circ} \mathrm{C}$. 


\begin{tabular}{l|lr|ll|ll} 
& ISRA (India) & $=\mathbf{1 . 3 4 4}$ & SIS (USA) & $=\mathbf{0 . 9 1 2}$ & ICV (Poland) & $=\mathbf{6 . 6 3 0}$ \\
Impact Factor: & ISI (Dubai, UAE) $=\mathbf{0 . 8 2 9}$ & PUHL (Russia) $=\mathbf{0 . 2 0 7}$ & PIF (India) & $=\mathbf{1 . 9 4 0}$ \\
& GIF (Australia) & $\mathbf{0 . 5 6 4}$ & ESJI (KZ) & $=\mathbf{3 . 8 6 0}$ & IBI (India) & $\mathbf{4 . 2 6 0}$ \\
& JIF & $=\mathbf{1 . 5 0 0}$ & SJIF (Morocco) & $=\mathbf{2 . 0 3 1}$ & & \\
\hline
\end{tabular}

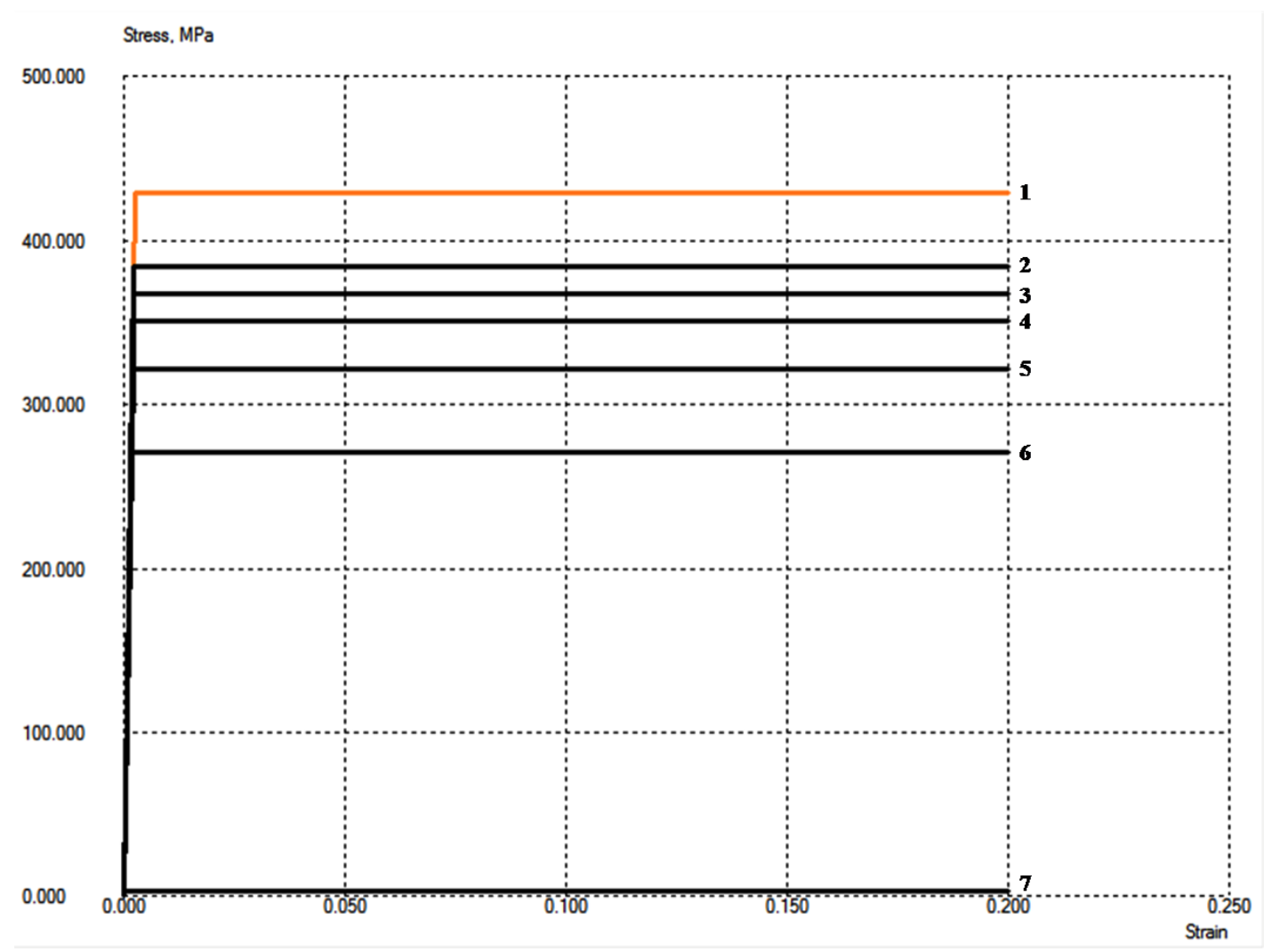

Figure 5 - The dependencies of stress in malleable cast iron EN-GJS-700 from deformation: 1) $\mathrm{T}=20^{\circ} \mathrm{C}, 2$ ) $\left.\left.\left.\left.\left.\mathrm{T}=100{ }^{\circ} \mathrm{C}, 3\right) \mathrm{T}=200{ }^{\circ} \mathrm{C}, 4\right) \mathrm{T}=300{ }^{\circ} \mathrm{C}, 5\right) \mathrm{T}=400{ }^{\circ} \mathrm{C}, 6\right) \mathrm{T}=500{ }^{\circ} \mathrm{C}, 7\right) \mathrm{T}=1153{ }^{\circ} \mathrm{C}$.

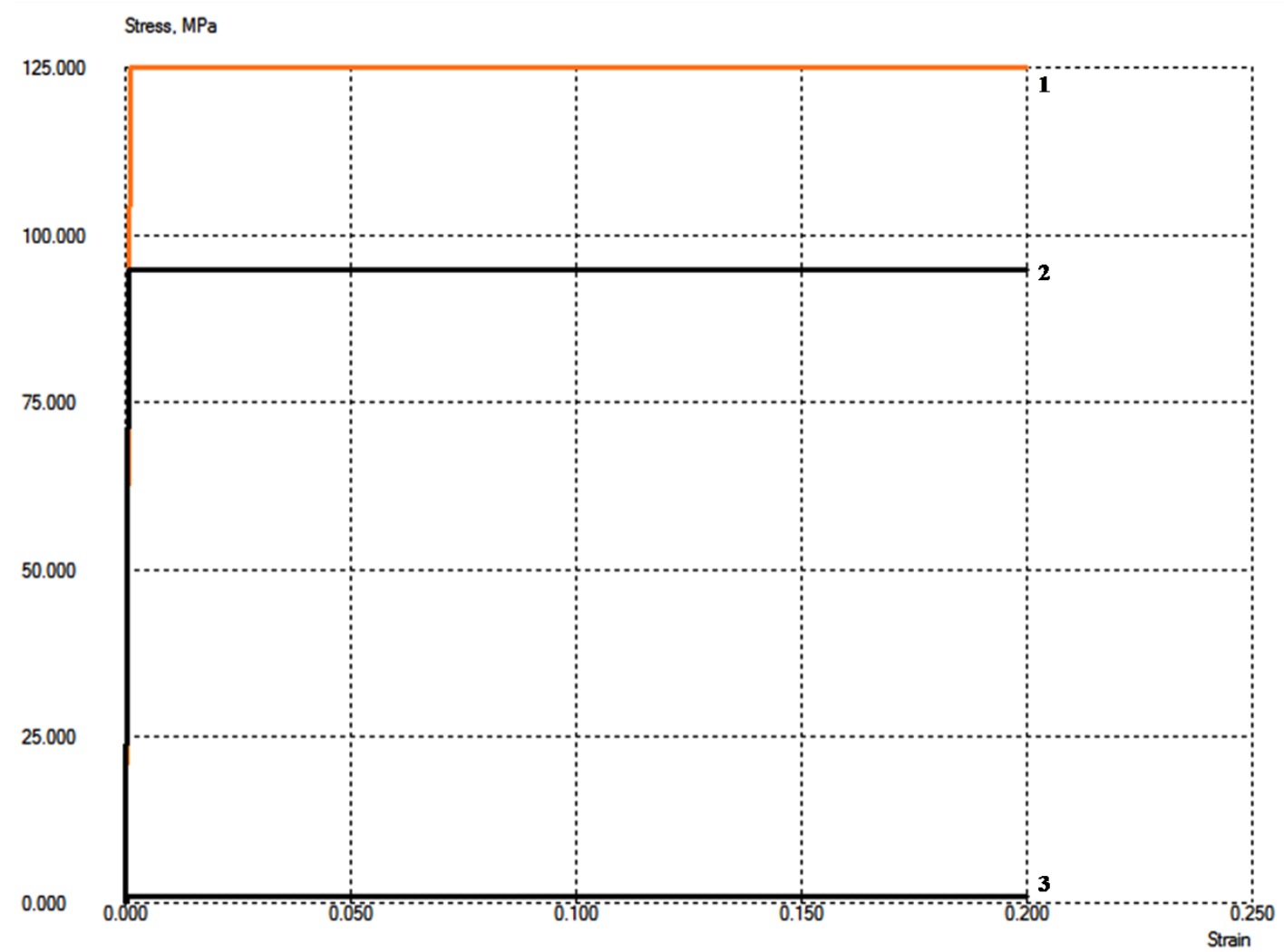

Figure 6 - The dependencies of stress in brass CuZn38Pb1.5 from deformation: 1) $\left.\mathrm{T}=100{ }^{\circ} \mathrm{C}, 2\right) \mathrm{T}=300{ }^{\circ} \mathrm{C}$, 3) $\mathrm{T}=925^{\circ} \mathrm{C}$. 


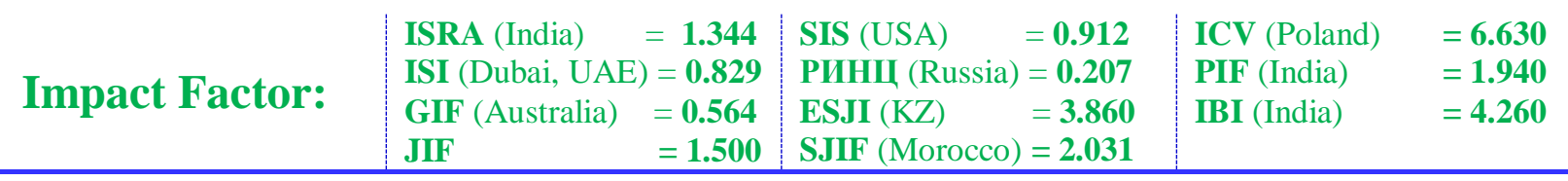

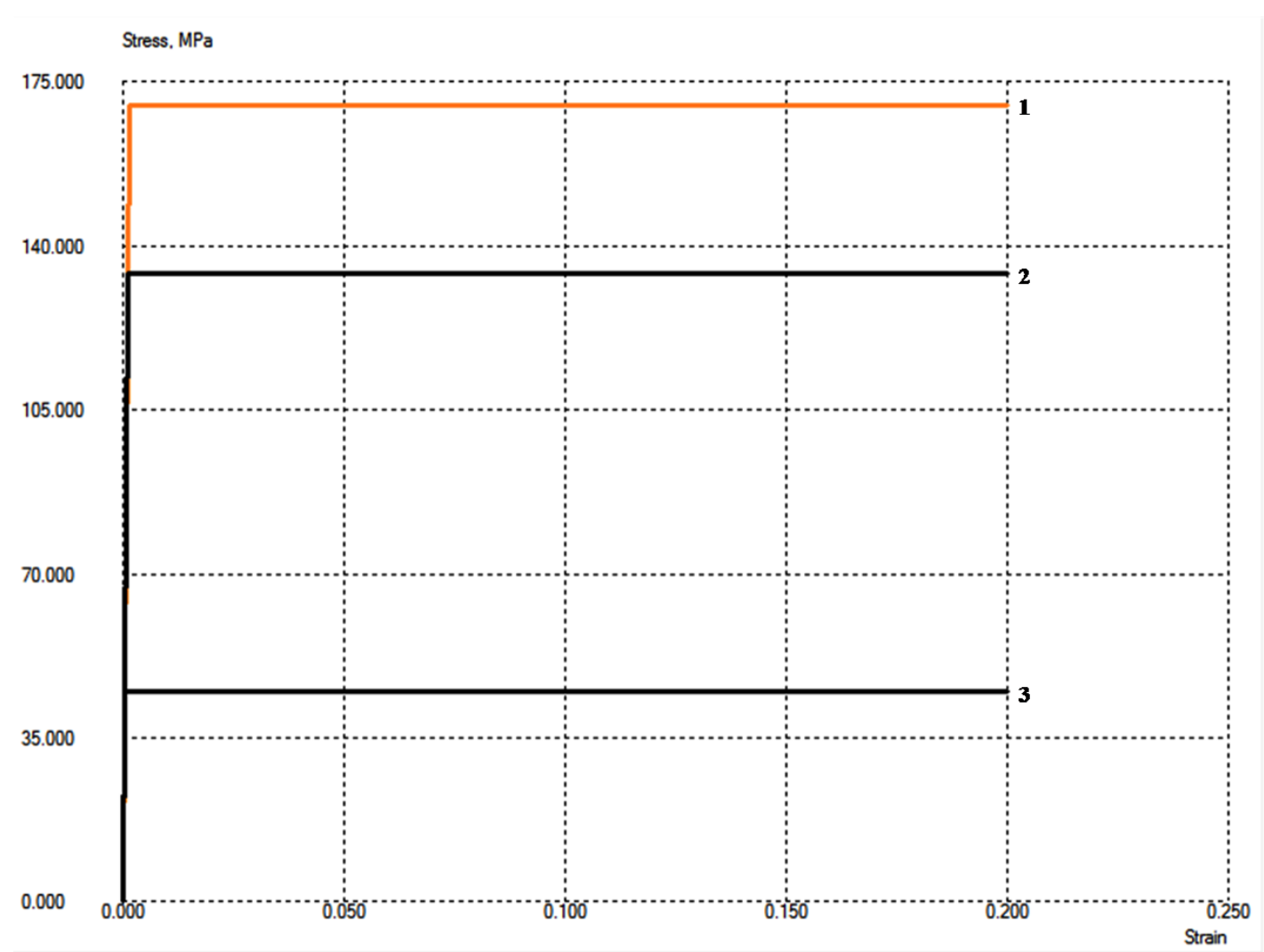

Figure 7 - The dependencies of stress in without tin bronze CC330G from deformation: 1) $\left.T=100{ }^{\circ} \mathrm{C}, 2\right) \mathrm{T}=$ $\left.300{ }^{\circ} \mathrm{C}, 3\right) \mathrm{T}=800^{\circ} \mathrm{C}$.

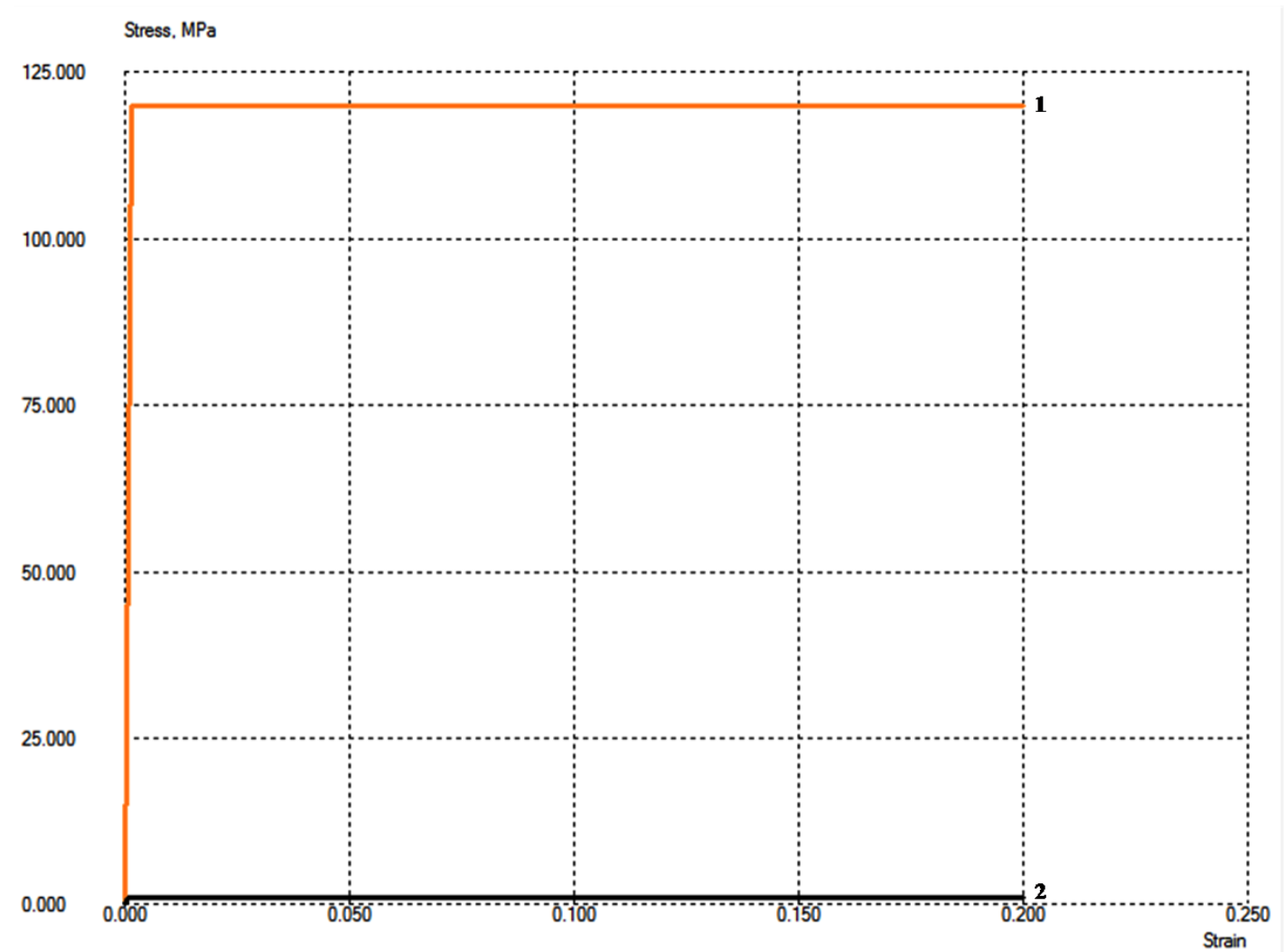

Figure 8 - The dependencies of stress in aluminium foundry alloy G-AlSi11 from deformation: 1) $T=100$ $\left.{ }^{\circ} \mathrm{C}, 2\right) \mathbf{T}=590{ }^{\circ} \mathrm{C}$. 


\begin{tabular}{l|lr|ll|ll} 
& ISRA (India) & $=\mathbf{1 . 3 4 4}$ & SIS (USA) & $=\mathbf{0 . 9 1 2}$ & ICV (Poland) & $=\mathbf{6 . 6 3 0}$ \\
Impact Factor: & ISI (Dubai, UAE) $=\mathbf{0 . 8 2 9}$ & PUHЦ (Russia) $=\mathbf{0 . 2 0 7}$ & PIF (India) & $=\mathbf{1 . 9 4 0}$ \\
& GIF (Australia) & $\mathbf{0 . 5 6 4}$ & ESJI (KZ) & $=\mathbf{3 . 8 6 0}$ & IBI (India) & $\mathbf{= 4 . 2 6 0}$ \\
& JIF & $=\mathbf{1 . 5 0 0}$ & SJIF (Morocco) & $\mathbf{2 . 0 3 1}$ & & \\
\hline
\end{tabular}

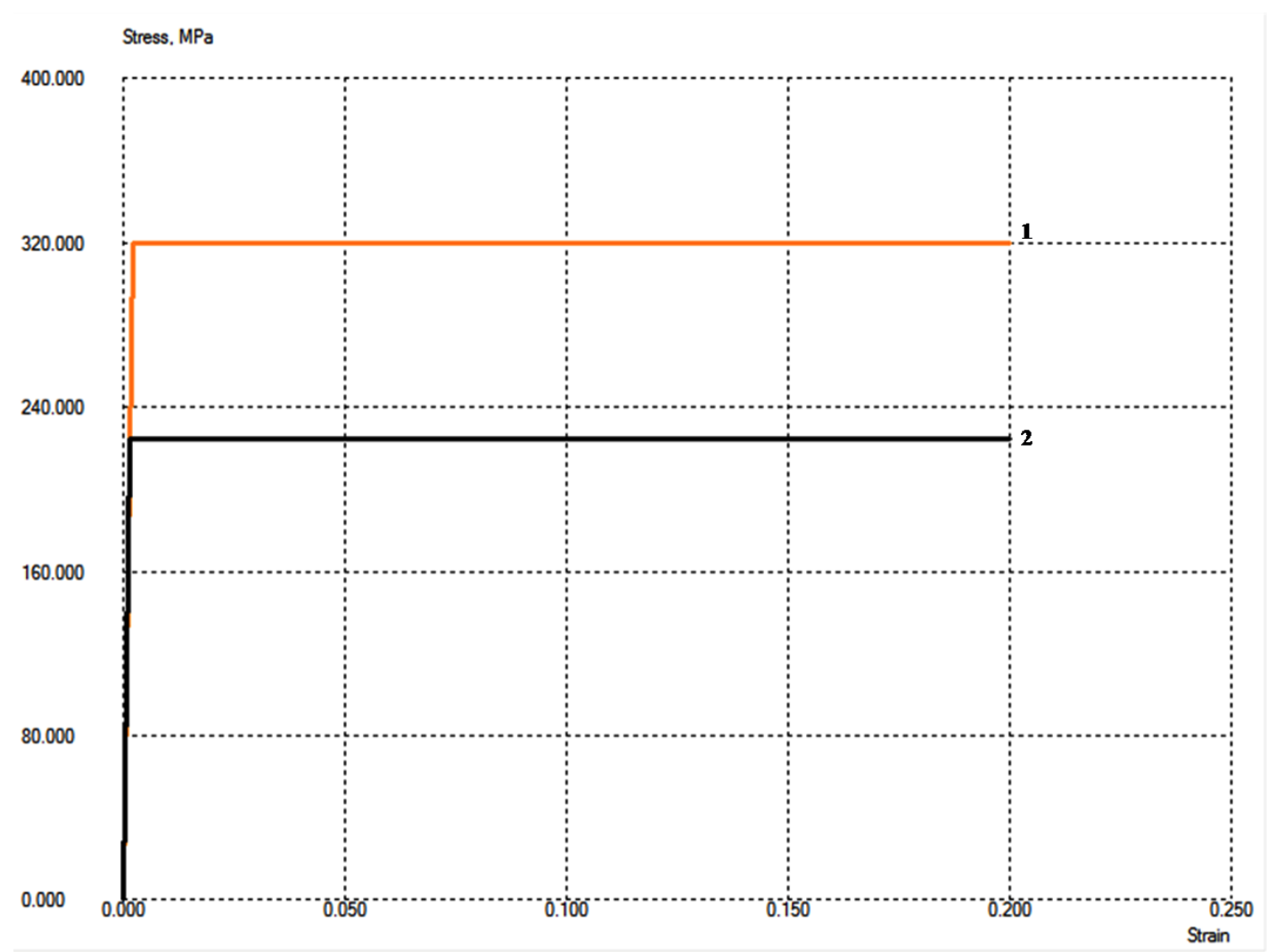

Figure 9 - The dependencies of stress in zinc alloy ZA-12 from deformation: 1) $\left.\mathrm{T}=100{ }^{\circ} \mathrm{C}, 2\right) \mathrm{T}=200^{\circ} \mathrm{C}$.

It is defined that alloy steel SCMnCr3 and corrosion-resistant steel X10CrNiTi18-9 are exposed to less stress than carbon steel $16 \mathrm{MnCr} 5$ at appropriate temperatures. Deformation of malleable cast iron EN-GJS-700 at a temperature of $100{ }^{\circ} \mathrm{C}$ is accompanied by the appearance of the stress value of $380 \mathrm{MPa}$. This is two times more than stress for corrosion-resistant steel X10CrNiTi18-9, and three times more than stress for gray cast iron EN-GJL200. For non-ferrous alloys same stress at a temperature of $100{ }^{\circ} \mathrm{C}$ is observed in brass $\mathrm{CuZn38Pb1.5}$ and aluminum foundry alloy GAlSi11. In zinc alloy ZA-12 in conditions of plastic deformation occurs stress which exceeds stresses of all non-ferrous alloys. Maximum stress in carbon steel $16 \mathrm{MnCr} 5$ has been calculated when deformation is more than 0.07. Maximum value of stress for all other alloys is observed when deformation is less than 0.05 .

\section{Conclusion}

Given the results of the research, highest stress occurs in the deformed machine parts made of carbon steel $16 \mathrm{MnCr} 5$. With increasing of thermal load, stress of alloy reaches maximum at a higher value of the coefficient of plastic deformation. The least stress occurs in the deformed machine parts made of grey cast iron EN-GJL-200.

\section{References:}

1. (2017) Elastic/Plastic Deformation. Available: http://www.ndeed.org/EducationResources/CommunityCollege /Materials/Structure/deformation.php (Accessed: 16.08.2017).

2. Volegov PS, Gribov DS, Trusov PV (2015) Damage and fracture: review of experimental studies. Physical Mesomechanics, №3. - pp. 11 $-24$.

3. Sangid MD (2013) The physics of fatigue crack initiation. Int. J. Fatigue, V. 57. - pp. 58 - 72.

4. Yoshida S, Toyooka S (2001) Field theoretical interpretation on dynamic of plastic deformation. J. Phys. Condens. Matter, V. 13. pp. $6741-6757$. 


\begin{tabular}{l|lr|ll|ll} 
& ISRA (India) & $=\mathbf{1 . 3 4 4}$ & SIS (USA) & $=\mathbf{0 . 9 1 2}$ & ICV (Poland) & $=\mathbf{6 . 6 3 0}$ \\
Impact Factor: & ISI (Dubai, UAE) $=\mathbf{0 . 8 2 9}$ & PUHЦ (Russia) $=\mathbf{0 . 2 3 4}$ & PIF (India) & $=\mathbf{1 . 9 4 0}$ \\
& GIF (Australia) & $\mathbf{0 . 5 6 4}$ & ESJI (KZ) & $=3.860$ & IBI (India) & $=\mathbf{4 . 2 6 0}$ \\
& JIF & $=\mathbf{1 . 5 0 0}$ & SJIF (Morocco) & $=\mathbf{2 . 0 3 1}$ & & \\
\hline
\end{tabular}

5. Tasan CC, Hoefnagels JPM, Diehl M, Yan D, Roters F, Raabe D (2014) Strain localization and damage in dual phase steels investigated by coupled in-situ deformation experiments and crystal plasticity simulations. Int. J. Plasticity, V. 63. - pp. $198-210$.

6. Lapovok R (2002) Damage evolution under severe plastic deformation. Int. J. Fract, V. 115. - pp. $159-172$.
7. Taheri S, Vincent L, Le-Roux J-C (2015) Classification of metallic alloys for fatigue damage accumulation: A conservative model under strain control for 304 stainless steels. Int. J. Fatigue, V. 70. - pp. $73-84$.

8. Chemezov D (2017) Shrinkage of some metal alloys after solidification. ISJ Theoretical \& Applied Science, 06 (50): 87 - 89. Soi: http://so-i.org/1.1/TAS-06-50-10

Doi: https://dx.doi.org/10.15863/TAS.2017.06.50.10 